\title{
Un piccolo sguardo oltre la scuola di specializzazione: l'esperienza di una giovane nefrologa
}

\section{Carmela Caputo}

\author{
U.O.S. Nefrologia e Dialisi, Ospedale S. Maria Nuova, Firenze
}

\begin{abstract}
A LOOK BEYOND THE RESIDENCY PROGRAM: A YOUNG NEPHROLOGIST'S WORKING EXPERIENCE
Abstract: A young nephrologist reports the experience of her post-residency carrier to give some food for thought and a practical help to the new class of young professionals. The young nephrologist may work in various areas that may differ from the school of origin in terms of viewpoints as well as for their cultural and practical aspects. Nephrology is actually a branch of Internal Medicine and includes different sub-specializations, such as peritoneal dialysis, hemodialysis, and renal transplantation. More often the young specialists work in areas in which, despite having the appropriate theoretical background, they do not yet have the practical experience required.
\end{abstract}

Key words: Young nephrologist, Fellowship, Practical experience

Conflict of interest: None.

Financial support: None.

Accettato: 5 Maggio 2014

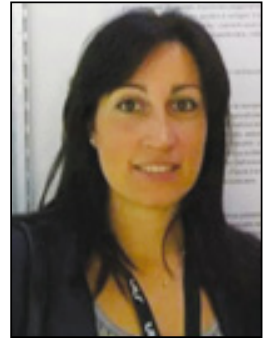

Carmela Caputo
Il mio percorso lavorativo è iniziato presso la scuola di specializzazione di nefrologia dell'Università Federico II di Napoli e si è svolto soprattutto nell'ambulatorio trapianto renale, dove, sotto la guida del Dr. R. Gallo e del Prof. M. Sabbatini, ho seguito per cinque anni circa 700 pazienti nel follow-up post-trapianto. Inoltre, mi sono in parte occupata dei pazienti, nell'immediato decorso post-trapianto, nel reparto sub-intensivo e di chirurgia dei trapianti. Essendo anche appassionata di medicina d'urgenza ho frequentato saltuariamente il reparto di Pronto Soccorso e di medicina d'urgenza dell'ospedale San Paolo di Napoli, diretto dal Prof. F. Schiraldi.

Il nefrologo che ha in carico i pazienti portatori di trapianto di rene è consapevole che sono pazienti complessi, perché esposti a una serie di patologie internistiche di tipo infettivo, dermatologico, oncologico, cardiologico o metabolico, a seguito anche del trattamento immunosoppressivo. Pertanto, durante gli anni di specializzazione, mi sono ritrovata a studiare, a osservare e a trattare in modo pratico queste molteplici patologie incidenti nel paziente sottoposto a trapianto di rene. Di conseguenza, mi sono portata dietro un bagaglio di nozioni utili per occuparmi non solo di pazienti trapiantati, ma anche di pazienti complessi di interesse internistico. Per quanto molti giovani, diventati ormai specialisti, vorrebbero continuare il percorso iniziato in specializzazione (per esempio, nel mio caso, mi sarebbe piaciuto continuare a occuparmi dei pazienti trapiantati), ciascuno di noi deve scontrarsi con la realtà lavorativa, che, in alcune regioni italiane più di altre, può essere più complicata e mettere alla prova "l'arte di arrangiarsi”, stimolando l'entusiasmo giovanile. Inizialmente è stata dura, poiché mi sono trovata a lavorare in centri di dialisi convenzionati, in un Pronto Soccorso di primo livello in provincia di Napoli e in un reparto di medicina interna. La mia scuola di specializzazione mi ha lasciato una solida base, sia nefrologica che internistica, grazie anche ai turni di guardia notturni, durante i quali mi ero spesso trovata a risolvere molteplici problematiche cliniche difficili, opinione peraltro condivisa da numerosi colleghi specializzandi. Dopo poco più di due anni di lavoro nei centri di dialisi privati ed essendo ancora off-limits l'accesso alla sanità pubblica in Campania, ho firmato il mio primo contratto a tempo determinato di tre anni nel ponente ligure, presso la Nefrologia e Dialisi dell'Ospedale di Albenga (SV), diretta dal Dr. G. Mancuso. Lì, finalmente, mi sono potuta di nuovo dedicare principalmente alla mia passione: il follow-up dei pazienti nel pre- e nel post-trapianto, oltre a continuare la mia esperienza in dialisi e negli ambulatori nefrologici. In Liguria ho lavorato per circa quattro anni 
con tre colleghi, in un reparto organizzato ottimamente, e ho potuto contribuire concretamente al potenziamento dell'attività trapiantologica del centro grazie alle mie conoscenze di base e alla mia esperienza napoletana. Insieme alla mia giovane collega Emanuela Vigo, siamo riuscite in quattro anni a portare al trapianto renale circa venti pazienti e a dare una speranza a più del doppio inserendoli in lista d'attesa di trapianto. Attualmente, sono circa 35 i pazienti trapiantati che sono seguiti periodicamente in regime ambulatoriale Posso definire un piccolo paradiso il reparto di Nefrologia e Dialisi di Albenga, un ambiente unito e gradevole con un'invidiabile collaborazione tra medici e infermieri e, non ultimo, un ottimo rapporto con i pazienti.

Ricordo con gioia i momenti condivisi con i pazienti: sia le gite semestrali, sotto la guida del Prof. R. Tassara, sia le importanti riunioni periodiche che trattavano argomenti come il trapianto renale da vivente, gli accessi vascolari per la dialisi e la terapia nutrizionale e che spesso si concludevano con note ludiche (ad esempio l'organizzazione del pranzo di Natale o delle prossime gite). Ad Albenga, non erano mai stati organizzati eventi per la prevenzione delle malattie renali sino al 2010, per cui diedi un mio contributo da coordinatore organizzando in prima persona la Giornata Mondiale del Rene (GMR) tramite il progetto Camper nella Piazza San Michele, nel 2010, e attraverso il progetto Scuole in un liceo scientifico, nel 2012.

Da un anno mi sono trasferita per motivi personali a Firenze nel reparto di Nefrologia e Dialisi dell'Ospedale S. Maria Nuova diretto dalla Dr.ssa F. Manescalchi.

Anche nella realtà fiorentina, grazie ancora al mio background acquisito dal mio Prof. V.E. Andreucci, uno dei primi promotori della GMR tramite la Fondazione Italiana del Rene, quest'anno ho organizzato il Progetto Scuola della GMR presso il liceo Galileo e, una settimana prima, una conferenza nell'Aula Magna dello stesso liceo, al fine di informare i giovani studenti sull'importanza sia della prevenzione delle malattie renali mediante un corretto stile di vita che della donazione degli organi.

In questa realtà lavorativa fiorentina, mi sto dedicando anche a inserimento, tunnellizzazione e rimozione di cateteri venosi centrali, pratiche svolte da me poche volte in precedenza, sia perché spesso affidate principalmente ai radiologi interventisti sia perché, negli anni da specializzanda, non era previsto un percorso formativo in tal senso. Di recente, mi sto occupando di ecografia renale e di accessi vascolari.

Ritengo che l'Università italiana formi bene i suoi medici. In particolare, la scuola di specializzazione di nefrologia attualmente diretta dal Prof. S. Federico mi ha lasciato solide basi teoriche, necessarie per contribuire in modo valido alle varie realtà nefrologiche che ho conosciuto.

Convinta che una parte molto importante della crescita professionale sia affidata anche all'entusiasmo, alla curiosità e allo spirito di iniziativa che ancora di più abbiamo noi giovani nefrologi, d'altra parte sarebbe opportuno migliorare e integrare la formazione dei neo-specialisti con un percorso che permetta l'acquisizione sia di competenze, ormai fondamentali, in merito all'inserimento dei cateteri venosi centrali e all'ecografia nefrologica e vascolare sia di tecniche come la biopsia renale e il confezionamento della fistola artero-venosa.

Molti giovani come me, terminata la scuola di specializzazione, partecipano a diversi corsi di approfondimento sulle tematiche succitate, anche se costosi, per completare la loro formazione. Sarebbe auspicabile iniziare questo percorso durante la specializzazione e, nei primi anni post-specializzazione, anche con il supporto della Società Italiana di Nefrologia (SIN), mediante l'organizzazione di corsi certificati per agevolare il percorso dei giovani nefrologi e favorire il loro adeguato inserimento nel mondo del lavoro.

In questi anni, la SIN ha dato un reale e forte contributo a noi giovani con il suo programma di fellowship nelle varie realtà nefrologiche italiane. Un'idea potrebbe essere quella di ampliare il programma anche nei centri europei di eccellenza. Certo, le diverse realtà nefrologiche italiane hanno un personale medico spesso in carenza, per il carico di lavoro, quindi potrebbe anche essere utile promuovere programmi di scambio lavorativo dei giovani nefrologi tra le varie strutture ospedaliere e universitarie, anche se per brevi periodi, perché, dal confronto e dalla condivisione di idee ed esperienze, la nefrologia italiana del futuro potrà sempre più crescere per dare un contributo di qualitá ai pazienti, come hanno fatto in modo eccellente i nostri maestri.

\section{Riassunto}

Una giovane nefrologa racconta l'esperienza decennale del suo percorso lavorativo per dare alcuni spunti di riflessione e piccoli aiuti concreti alla nuova classe di giovani professionisti, che potrebbero ritrovarsi a esercitare in realtà diverse dal punto di vista organizzativo, culturale e pratico rispetto a quella della scuola di specializzazione di provenienza. La nefrologia è una branca specialistica della medicina interna che a sua volta racchiude in sé diverse ultraspecializzazioni, come la dialisi peritoneale, l'emodialisi extracorporea, il trapianto renale e così via. E, quindi, concreta la possibilità che i neospecialisti si trovino a lavorare in settori in cui, pur avendo le appropriate conoscenze teoriche, non hanno ancora la dovuta esperienza pratica.

Parole chiave: Giovani nefrologi, Fellowship, Esperienza pratica

Dichiarazione di conflitto di interessi: L'Autore dichiara di non avere conflitto di interessi.

Contributi economici agli Autori: L'Autore dichiara di non aver ricevuto sponsorizzazioni economiche per la preparazione dell'articolo.

Indirizzo degli Autori:

Dr.ssa Carmela Caputo

U.O. Nefrologia e Dialisi

Ospedale S. Maria Nuova

Piazza S. Maria Nuova 1

50122 Firenze

nefrolina74@gmail.com

carmela.caputo@asf.toscana.it 